"Doing my profession is also part of worship": How Clinical Psychologists Address Aspects of

\title{
Spirituality and Religion in Indonesia
}

\begin{abstract}
Andrian Liem
School of Psychology, the University of Queensland, Australia; andrian.liem@uq.net.au
\end{abstract}

\begin{abstract}
This study aimed to explore how Indonesian clinical psychologists (CPs) address aspects of spirituality and religion (SR), particularly their attitudes towards and experience of it, on the mental health context. Semi-structured interviews were conducted with 43 CPs in public health centres in Yogyakarta Province, Indonesia. Data were anyalsed using deductive thematic analysis and they generated ten sub-themes which were merged into three central themes. The first theme was experiences related to SR, particularly in Indonesian sociocultural context. The second theme concentrated on participants' clinical experience related to SR integration into clinical practice. The last theme highlighted the effort made by participants to create holistic mental health services. The originality of this study was represented by the interview quote in the title, "Doing my profession is also part of worship". It was found that SR is part of culture and belief among Indonesian people, including CPs and mental health treatment clients. In summary, participants genuinely acknowledged that they were not able to completely detach SR from their professional practice. However, participants also pointed out that they were different with spiritual-religious healers (SRHs) and favourably welcomed future collaboration with credible SRHs. This positive attitude embodied a holistic care approach that recognises the diverse biopsycho-social-spiritual needs of clients. Therefore, professional organisations and psychology faculties should establish regulations and education of SR in psychology curricula and conventional psychotherapy to achieve this holistic mental health services in Indonesia.
\end{abstract}

Keywords: clinical psychology; cultural psychology; ethic and professionalism; holistic medicine; qualitative methods 


\section{Introduction}

Originally before the $19^{\text {th }}$ century healthcare facilities incorporated spirituality and religion (SR) aspects in clinical practice, including for mental health treatments (Oxhandler \& Parrish, 2018). However, SR was gradually rejected in the psychology discipline in the early 1900s when pioneers of psychotherapy, such as Freud, criticised religion as a universal neurosis (Hofmann \& Walach, 2011). Moreover, SR was completely discarded and ignored from mental health treatment when behaviourism and cognitivism blossomed between the 1920s and 1980s (Barnett \& Johnson, 2011). Therefore, clients often chose to suppress their spiritual or religious beliefs out of fear of being pathologised by mental health professionals as explained by South African psychologists (Brown, Elkonin, \& Naicker, 2013).

\section{What is spirituality and religion?}

Numerous definitions and understandings of spirituality and religion have been developed since its revival among mental health professionals in the 1990s (Patel \& Shikongo, 2006). Generally, spirituality is understood as a transcendent personal experience and religiosity as a communal-denominational worship ritual (Harris, Randolph, \& Gordon, 2016; Passalacqua \& Cervantes, 2008; Shafranske \& Cummings, 2013). The connection between these two terms is complex because they encompass unique yet overlying dimensions; for example, an individual may be religious, spiritual, or both (Barnett \& Johnson, 2011; Frazier \& Hansen, 2009; Hofmann \& Walach, 2011). Therefore, in the present study these terms are used conjointly unless a specific term is needed to explain a particular context.

Other terms used in this study are spiritual-religious healers (SRHs) and spiritualreligious therapy (SRT). The SRHs term covers all non-conventional health professionals who use SR in their practice. Specific terms such as 'spiritual healer' are used when describing a contextual situation. Additionally, a spiritual healer may be referred to within 
specific cultures by a range of indigenous names (Liem \& Rahmawati, 2017), e.g. balian (Balinese spiritual healer) or dukun (a term used across many areas of Indonesia, referring to indigenous healers of both a physical and spiritual nature). Meanwhile, the term 'religious healer' is used to cover clergy and religious leaders without referring to a particular religion. The SRT term encompasses therapy, practiced by SRHs or conventional health professionals that contains any spiritual-religious philosophy, teaching, or activity in the process.

\section{The holistic approach}

The multidiscipline collaboration in contemporary psychiatric services has seen SR being included more often as part of a holistic care approach (Ramakrishnan et al., 2015). This approach emphasises the wholeness and multidimensional needs of clients and their families (Rochmawati, Wiechula, \& Cameron, 2018). In this approach, the interrelation of a client's mind, body and spirit is recognised by acknowledging biopsycho-spirit-sociocultural factors that influence a client's condition (Brown et al., 2013; Suryani, Lesmana, \& Tiliopoulos, 2011). Previous studies found that addressing or integrating SR into mental health services had significantly increased clients' coping skills, resilience, and well-being (Chaudhry, 2008; Kalra, Shah, Deakin, \& Bhugra, 2015); and helped clients with posttraumatic stress disorder in renewing hope and re-finding life purposes (Harris et al., 2016; Rochmawati et al., 2018).

These positive outcomes have led to prolific quantitative studies to survey mental health professionals' attitudes towards SR in secular nations. The results showed that psychologists in the USA (Shafranske \& Cummings, 2013; Shafranske \& Malony, 1990), UK (Crossley \& Salter, 2005), and Germany (Hofmann \& Walach, 2011) had positive attitudes towards SR and some of them had integrated SR into their therapy. Additionally, psychologists in the USA, Canada, Australia, and New Zealand self-reported for being more spiritual than religious (Delaney, Miller, \& Bisonó, 2007; Harris et al., 2016). However, one 
study in the USA also showed that psychologists had the lowest positive attitudes towards SR and the fewest professional courses about SR when compared with nurses, social workers, counsellors, and marriage-family therapists (Oxhandler \& Parrish, 2018). These four professions had been prepared better than psychologists to integrate SR into their practice through cultural competency training, including SR aspects, in their professional education (Barnett \& Johnson, 2011).

\section{The current study and SR in Indonesia}

Despite abundant surveys of psychologists' attitudes towards SR, how psychologists address SR in their practice is the most under-examined topic in this area (Crossley \& Salter, 2005). Also, the results of study in developed-secular nations may be different compared with those in developing or non-secular nations due to sociocultural variations (Chaudhry, 2008; Javed, 2015). Therefore, the current study aims to explore how Indonesian clinical psychologists (CPs) address aspects of SR, particularly their attitudes towards and experience of it, in a mental health context using qualitative methods. This exploratory study was part of a national survey of complementary-alternative medicines (CAM) among Indonesian CPs using a mixed-method design. From the nationwide survey, CPs in Indonesia reported that

SRT was the most commonly used CAM method both for personal and professional purposes (Liem \& Newcombe, 2019).

Indonesia is unique because it is neither a secular nor a religious nation, represented by the nation's official "Five Founding Principles" (known as the Pancasila); the first of which states that Indonesia is founded on the "belief in one supreme God" ("Ketuhanan Yang Maha Esa”) (Ropi, 2017). Additionally, there are six recognised religions and more than 100 aliran kepercayaan (traditional faith) in Indonesia, though the population is predominately (87\%) Muslim (Rochmawati et al., 2018). However, the percentage of population depends on the 
local culture and area; for instance, in Bali more than $83 \%$ adhere to Balinese Hinduism which differs from Hinduism in India (Suryani et al., 2011).

A national survey conducted by the Indonesian Ministry of Health discovered that more than $67 \%$ of people in Indonesia came to SRHs because of their tradition/culture, felt it was more effective, and felt conventional medicine was hopeless (Kementerian Kesehatan RI, 2013). Therefore, a holistic care approach was particularly important for mental health treatment in nations with strong SR influence such as Indonesia (Suryani et al., 2011). Previous studies among health professionals in Indonesia showed Indonesian physicians (predominantly Muslims) were significantly more religious, more spiritual as well as more willing to integrate SR into their practices than Indian physicians (predominantly Hindu) (Ramakrishnan et al., 2015); palliative care staff in Indonesia also provided SR support to their clients and families, i.e. by praying together, which was perceived as culturally contextual and may be controversial in secular nations (Rochmawati et al., 2018).

\section{Methodology}

Data were collected through semi-structured, face-to-face interviews using an interview schedule revised based on the pilot interview (Liem, 2018). This study had been reviewed and granted ethics approval by the Ethics Committee of School of Psychology at the University of Queensland. The sampling for maximum variation was used in this study to recruit CPs in all 43 PHCs from two districts in Yogyakarta Special Region Province, Indonesia. The only inclusion criterion applied was "Participant is willing and able to give informed consent for participation in the study."

\section{Procedure and participants}

Interviews were conducted by the first author between November 2016 and January 2017 in two sub-districts of Yogyakarta Special Province: Yogyakarta City and Sleman. 
127

128

129

130

131

132

133

134

These two of five sub-districts were chosen because the integration of psychological services

\section{into public health centres in Indonesia were initiated here (Setiyawati, Blashki, Wraith,}

Colucci, \& Minas, 2014). Before having the face-to-face interview, participants were given a chance to ask questions related to the research and asked to sign the consent form. All participants voluntarily agreed to be interviewed and audio-recorded at their suggested time and place. Each interview lasted for between 30 and 100 minutes, with an average of 55 minutes. Participants ranged from 25 to 42 years old with an average age of 34 years old and they were from Java, Sumatra, and Borneo Island. There was only one male participant and therefore 'she' is used to discuss all interview responses in this study to maintain participants' anonymity.

\section{Interview schedule}

An interview schedule (a guideline for conducting interview) was used because the interviewer has more flexibility, compared with interview protocol, when using it as a guideline (Bantjes \& Van Ommen, 2008; Dikko, 2016). Three areas of SR were explored among participants through interview schedule. The first area was participants' experiences of spirituality and religion in both personal and professional settings. The second area of interview focused on participants' attitudes towards SRHs. The last area explored participants' attitudes towards SRTs, especially related to its integration into psychological services. The results of this interview schedule development and pilot study had been reported elsewhere (Liem, 2018).

\section{Data analysis}

Interview transcripts were analysed using thematic analysis due to its flexibility to both report and examine explicit and latent contents (Braun \& Clarke, 2006). Deductive thematic 
152

153

154

155

156

157

158

159

160

161

162

163

164

165

166

167

168

analysis (Guest, MacQueen, \& Namey, 2012) was particularly used because this follow-up qualitative study aimed to explain specific quantitative findings. The steps of analysis followed guidelines from previous studies (Braun \& Clarke, 2006; Guest et al., 2012). The data analysis was then undertaken based on the checklist for reporting qualitative health research (Tong, Sainsbury, \& Craig, 2007) to improve the report's quality.

\section{Trustworthiness}

The trustworthiness of this qualitative study was ensure principally through the selection of deductive thematic analysis where the author recognised the influence of previous studies in developing this current research. The researcher (the author) acknowledged that researchers cannot completely free themselves from a theoretical framework (Braun \& Clarke, 2006). To increase the credibility, all of the interviews were conducted by the same interviewer (the author) and all of the transcripts were double-checked by comparing them with the audio recordings. Lastly, the author consulted with a senior lecturer of Indonesian study who is also an expert in qualitative methodology to improve the conformability of the analysis and interpretation (Smith \& Wu, 2012; Vaismoradi, Turunen, \& Bondas, 2013). 


\section{Results}

A thematic analysis of the 43 interviews generated ten sub-themes which were merged into three central themes which is presented in Figure 1. The first theme focused on clients' and participants' experiences related to SR, particularly in their sociocultural context. The second theme concentrated on participants' clinical experiences related to SR integration. The last theme highlighted the efforts made by participants to create holistic mental health services. Extracts and quotes are presented by participant number and transcript line, for example, (28-120) means a transcript from Participant 28 on line 120.

\section{Unfolding clients' and clinical psychologists' stories}

This first theme — clients' and participants' experiences of SR — covered four subcentres. It was also found that family played a vital role in decision-making around mental health treatment for clients. In addition, SR as part of Indonesian cultural and belief systems could not be detached from both client's and clinical psychologist's lives. However, participants as professional helpers should be able to differentiate their practice from that of SRHs by relying on conventional psychotherapy processes. In the last sub-theme, it was explained why participants showed more favourable attitudes towards religious healers than spiritual healers.

The journey of clients and their families. Participants highlighted that understanding clients' behaviours, particularly the reason why they went to SRHs, is an essential foundation for comprehending SR in an Indonesian mental health context. Figure 2 displays participants' explanation of their clients' decision-making processes related to SRHs and conventional 
health services visits. Generally, clients visited SRHs in the first place because of several reasons. For example, clients from remote areas often went to SRHs because the location was closer than conventional health services. The cost included both the long distance and time taken to visit conventional health services; there was also potential financial loss because clients and their families in villages were usually paid daily for their labour. Therefore, if they must travel far away, they would receive no income for the day. Participants also observed that mental health information in remote or suburban areas was insufficient. Also, a client's parents who may be perceived as elders or authority figures in their area will most likely bring their child to a SRH first because they do not trust conventional health services. It was discovered that family members are often intensely involved in a client's mental health treatment. Participants explained that most parents of child clients, and husbands of female clients, often brought their child or wife to meet a SRH before finally coming to conventional health services. Extended family members, particularly in rural areas, could also be actively engaged in a client's treatment. For example, the family might bring the client to a SRH in one single meeting or leave them at pondok (inpatient SRT care), especially if the client has a psychotic disorder. Family members might leave the client at pondok because they do not have enough resources (time, money, and persons) to take care of the client at home (28-120).

Clients or their family members came to conventional health services, PHCs or hospitals, after they showed no progress or when their condition became worse. At the conventional health services, clients were prescribed medicines, particularly for severe mental disorders. Some family members brought clients back again to a SRH without telling the conventional health practitioners because they did not want the client to become dependent on drugs (41-48). Another reason for bringing a family member to a conventional health service was that they were only interested in methods that would have the fastest 
219 effect. Participant 19 added that if client could not do the rituals requested by the SRH, the

220 rituals still could be done by their family members, for example, praying at midnight. This flexibility in therapy, which could not be conducted in conventional medicine, additionally inspired the family to bring clients to SRHs. In some cases, clients might relapse because of numerous reasons and, when this happened, they preferred to go to the SRHs again instead of returning directly to conventional health services. In another scenario, some families might lock up or restrain clients at home and clients would be brought to conventional health service again after their condition was very severe or when their neighbours encouraged them to do so $(26-83)$.

Participants said that CPs might maximise this family involvement in conventional mental health care. For example, CPs can consistently educate the family about mental disorders and ask them to actively participate in a client's treatment. CPs might also organise family gatherings for clients with severe mental disorders. Some participants had applied this strategy and used the family gatherings to develop social support for clients' families as caregivers.

[Insert Figure 2]

SR as part of culture and belief. All participants stated that SR, including mystic and supernatural forms, is part of Indonesian culture and beliefs, and part of people's daily lives. Therefore, it is still common for Indonesian people, particularly in villages, to think that supernatural forces can cause physical illness and mental disorders. Participant 37 gave an example where one family insisted on bringing a client while being critically ill with dengue fever from a PHC to a SRH because the family believed that the cause of his illness was evil spirits in the room. Unfortunately, the client died on the way when his family was bringing him to the SRH in a different city. 

sides of the same coin. It may act as a protective factor by facilitating aspects of Indonesian collective culture and providing a space for individual needs. For example, sholat Jumu'ah (Friday prayer) in Islam and Sunday mass in Christianity are opportunities for community gatherings; sholat Tahajjud (late night prayer) and confession to a clergy member in Catholicism can be used for individual catharsis.

On the other side, SR can also be a risk factor for mental health treatment. For example, SR beliefs may maintain stigma towards people with mental disorders, making their family ashamed of the client and locking them up at home. Additionally, people may also be unfamiliar with the psychology profession and suspect psychotherapy contradicts religious teachings, as discussed by Participant 39 . This stigma and unfamiliarity possibly cause clients and their families to distrust CPs by not disclosing if they have visited SRHs. Participants also explained that CPs will never win when competing with SRHs so that it is better to make a dialogue with them as an ally. Hence, participants cannot apply psychotherapy rigidly as written in textbooks because they need to adjust their therapy methods according to local culture, as explained by Participant 38. Although, if a CP chooses not to integrate SRT into their practice, the CP must be aware of the client's SR beliefs so that the psychotherapy will not contradict these beliefs.

SR is also part of health professionals' lives in Indonesia, as participants shared numerous stories of their colleagues at their PHC. For instance, as told by Participant 19, nurses believed that hysteria was caused by bad spirits so that they invited SRH into the emergency room. As another example, some colleagues at the PHC asked participants about ruqyah (Islamic exorcism) practitioners because they believed that supernatural forces had caused the mental disorders experienced by their family members. Participants' colleagues 
the treatment administered there. Also, they felt ashamed and afraid that their co-workers would gossip about them.

Participants as people with religious faith also acknowledged that they could not be completely free from their religious values and personal bias. For example, they agreed that the primary mental health treatment should be conventional medicine through biopsychosocial intervention. However, they highlighted that it should also be complemented with spiritual efforts, such as praying. Also, participants believed in supernatural forces, including people with special gifts to communicate with creatures from different dimensions. Participant 32 mentioned that she could recognise the difference between clients experiencing clinical hallucinations and those who had this special gift. She admitted, however, that it was difficult to explain her observation scientifically.

Moreover, religion and science cannot be separated, as emphasised by Participant 12, “Doing my [CP] profession is also part of worship. I can't and wouldn't separate these two things." (12-70). This condition created a dilemma for some participants as exemplified, "In the professional oath we must be neutral, should not bring religion into it. But I found it is difficult to implement the oath in our religious society where SR is part of everyone's life." (1-48). Thus, participants strongly suggested that psychology faculties should address this issue in educational training and that professional organisations should establish a standard operating procedure (SOP) for SRT integration in psychotherapy.

What makes CP and SRH different? Apart from integral SR in participants' lives, many people recognised three aspects that differentiate participants and SRH when doing SRT, as presented in Table 1: assessment and treatment plans, intervention types, and communication style. Participants assess a client's core problems and what efforts the client has done, then writes a psychological assessment report. Based on the assessment results, they set up 
intervention goals and plan the treatment together with the client. SRT will be integrated into psychological treatment only if a client needs it. Participants will not use SRT if the client shows symptoms of having a severe mental disorder and directly refers them to hospital. On the other hand, participants perceived that SRHs do not have a framework to assess client's problem and plan the treatment based on their faith only.

For interventions, participants explained that if they use a SR activity, it has to be part of conventional psychotherapy. Participants will explain to clients the psychological benefits and scientific reasons for using the SR activity. Moreover, the SR activity which is integrated into the conventional psychotherapy should be a simple and basic one. For example, a CP may ask a client to do zikir (Islamic chanting) as part of a breathing relaxation technique. Also, participants will educate their clients that psychotherapy will not instantly solve their problems so that they must be patient and actively participate in the process.

SRHs, by contrast, will apply SRT to every client's situation, including for clients with psychotic symptoms. SRHs will also ask clients to read specific bible verses or to perform rituals to rid them of bad energy or evil spirits. In addition, SRHs tend to communicate with clients using dogmatic and doctrinal styles while participants apply micro-counselling skills and show empathy with their clients. For example, Participant 12 shared her story about an adolescent client who was sent by the client's teacher at an Islamic boarding school to a SRH because the client was experiencing a sexual identity crisis. Because of the client's religious background, she used Quranic chapters not for preaching but for explaining that the Prophet Muhammad's character was a gentle and loving man: this was used to help the client so that he needed to not worry about being a gentle and sensitive man.

\section{[insert Table 1]}


Participants' attitudes towards SRT and SRHs. Some participants expressed doubt towards the effectiveness of SRT conducted by SRHs, and even by participants' colleagues or senior CPs. Participants hardly ever found research articles to support the SRT used by them. Also, their colleagues or seniors did not explain clearly the scientific reasons of incorporating SRT into their practice. Hence, they assumed that SRT effectiveness might only be a placebo effect. However, participants accepted that their clients met SRHs in order to deal with a condition for which they also sought help from conventional health services. Participants requested that clients inform them about what interventions were given by SRHs, especially herbal remedies, to avoid adverse-reactions.

Participants generally showed more favourableness towards religious healers than spiritual healers. Participants perceived spiritual healers, and also some religious healers, performed methods and asked clients to do behaviours that deviate from religious teachings and that did not have scientific supports. In addition, participants questioned the efficacy and scientific logic of the methods. For example, one participant expressed her doubt about the efficacy of such methods when recounting a story of a spiritual healer who had applied chicken blood to the vagina of her client with a psychotic disorder because it was believed to remove her bad luck (24-96). In addition, participants also found that their clients spent a lot of money to pay and buy objects requested by the spiritual healers, as exemplified, "She spent millions, even borrow from the bank, for treatment with orang pintar [traditional healer] and buy requested stuffs like special oil." (43-64). In some cases, religious healers also did unscientific methods which were against conventional medicine practices. For example, as told by Participant 37, one religious healer performed ruqyah (Islamic exorcism) but, in the process, he spat in the client's face; he also stated that prescription drugs were toxic for the client's body and then ordered the client to stop taking them and instead to soak in the creek at midnight. 

incidents experienced by participants or their clients. For example, Participant 36 had a client with breast cancer who initially only came to her spiritual healer and was given prayer water.

The cancer became worse and made the client depressed so she went to the Participant.

However, it was too late because the cancer had reached the Stage 4 and finally the client died. Some methods performed by spiritual healers could also be categorised as sexually predatory behaviours. For example, Participant 29 said that her teenage client was manipulated by the spiritual healer into having sex with him and developed depression because she became pregnant. Knowing this story, the Participant told the client's parents and encouraged them to report the case to the police.

\section{Practical experiences from integrating aspects of SR in psychological services}

This second theme begins with the description on how far aspects of SR could be integrated into a CP's clinical practice. This is followed by participants' experiences of how they integrated SR into their practice. The efforts made by participants to improve their SRT knowledge and skills are presented in the last sub-theme.

\section{Boundaries and limitations of SRT integration into psychology practice. Participants}

indicated that the boundaries for how far SRT could be integrated into psychological intervention was marked by three sources: the client, the $\mathrm{CP}$, and the particular SR activity. SRT could be applied if it was needed by clients and they showed an interest in applying it as part of a particular intervention and their lives. Participant 37 emphasised that CPs cannot force clients to do SR techniques if the clients are not ready or willing to do it. Clients should also understand the religion and practice religious activities in their daily lives for SR to be an appropriate method. 
The SR activity to be integrated into the intervention should be understood comprehensively and practiced daily by the CP. This is important because CPs need to be able to explain it in details; when CPs practice the SR activity in their life, clients will build trust with the CPs. Nonetheless, participants also warned that CPs need to be careful with potential bias from their personal values and religious understanding. Therefore, CPs need to have specific SRT skills and competency to integrate SR methods into their conventional psychotherapy. Participant 41 highlighted that CPs must take part in SRT training which is accredited by psychology and medical professional organisations before they can integrate it into their practice. However, other participants expressed their concern about some CPs using $\mathrm{SR}$ in their practice; they viewed a $\mathrm{CP}$ using SR as performing a double-role, creating a challenge for the individual to draw a line between what they do as a $\mathrm{CP}$ and as a religious person or clergy member. For example, Participant 30 expressed her confusion about some colleagues and senior CPs who were also clergy members, commenting that they often integrated SR activities into their practice, even when it was not needed by their clients.

SR activity is often integrated into conventional psychotherapy and is expected to be part of basic practices and is not perceived to deviate from religious teachings. More complex or advanced SR activities, i.e. bible interpretation, should be conducted by a clergy member. Participants also emphasised that SR activities need to be connected with psychological theory and only be offered as a complement to conventional psychotherapy as exemplified by, "For example, cognitive or behaviour therapy has been given initially, and then we insert this [SR activity] as a complement." (43-74). Participants also underlined that SR activities based on scientific evidence should be integrated into psychotherapy.

Besides boundaries for when SRT could be integrated into psychological practice, participants also acknowledged the limitations of SRT. Based on participants' observations, SRT was not effective for clients with severe mental disorders because they might interpret 
religious teaching mistakenly or it was potentially worsening their condition. Participants strongly suggested limiting SRT integration only for clients with mild mental disorders and those only experiencing the stressors of daily hassles. Participants also concluded that SRT was not useful for children and adolescent clients. Clients of that developmental age have not developed a comprehensive understanding and interpretation of religious teachings so that it would be difficult for a CP to explain the benefit of SRT. In addition, participants said that children and adolescent clients were mostly experiencing pervasive developmental disorders such as autism which would be more effectively treated with conventional medicine rather than SRT.

Integration of SR into psychological practice. Participants integrated aspects of SR into their practices in two ways. First, participants talked about SR concepts, i.e. forgiveness, with clients showing an interest in SRT during the counselling process. Participants also often inserted SR concepts into family and marriage counselling because these are often also part of religious teachings. Second, some participants integrated SR activities as part of psychotherapy, especially for sleep disorders, anxiety, depression, for elderly clients, and for clients with chronic illnesses and those under palliative care. Participant 30 gave an example involving her religious client with post-traumatic stress disorder from sexual abuse. The client used to be a member of her church choir and the participant asked the client to try this activity again to rebuild her client's confidence and self-esteem. Participant 20 illustrated a different case where she had a client who insisted on asking for ruqyah (Islamic exorcism). The participant did not have the skills to perform a ruqyah so she invited her colleague who was also a CP and able to conduct the ruryah. The client felt better after the ruqyah and continued the conventional psychotherapy as planned. 
The majority of participants were Muslims and their clients were also predominantly

418

419

420

421

422

423

424

425

426

427

428

Muslim. Therefore, Islamic teachings and activities were the most common integrated religion into participants' practice, even by non-Muslim participants when explaining an activity's benefits and psychological effects to clients. However, Participant 19 highlighted that she, as a non-Muslim CP, did not give behavioural SRT, i.e. zikir (Islamic chanting), because it might be incorrect or make the client uncomfortable. On the other hand, Muslim participants described briefly particular Islamic teachings or activities and asked non-Muslim clients if there was any similar things in the client's own religion. Participants then encouraged clients to do the religious activity or discuss it with a member of the clergy from their religion. Both Muslim and non-Muslim participants agreed that there are universal values, such as compassion, in every religion's core belief system. These universal values might be called spirituality which has a more profound meaning than religion and thus can be incorporated into SRT with the many religions and faiths of clients.

Participants underlined the importance of assessment before integrating SR into psychological therapy because each client has a different level of interest in SR. Three methods were used to assess a client's interest towards SR integration: their outfit, content of the discussion, and the client's habit of practising religious activities. Clients were predominantly Muslim women wearing hijab so that it could be perceived as a sign that religion was important to them. However, not all clients with religious outfits were interested in integrating SR into their psychotherapy because they wanted more concrete action, as told by Participant 11. Clients who frequently initiated discussions about SR or who mentioned their habit of practising religious activities in their daily life were most likely to be interested in integrating SR into the psychotherapy process.

It was found that not all participants integrated aspects of SR into their practice because of three reasons. First, some participants considered SR beliefs belong to private domain and 
everyone has their own religious interpretation and faith. For example, Participant 41 explained that there are several streams within Islam itself and she was practising a nonpopular stream in Indonesia. Hence, even when she meets Muslim clients, they may have different belief systems and understandings of Islamic teachings. Second, some participants or their family members have had negative experiences with SRHs or SRT such as ruqyah (Islamic exorcism) and so they did not want clients to have the same experience. Lastly, participants acknowledged that they did not have the competency for conducting SRT so they preferred practising conventional psychotherapy techniques only.

\section{Recommendation and referral of SR. Some participants gave non-clinical} recommendations related to SR activities and explained the psychological benefits for them. For example, they recommended that Muslim clients should continue to do shalat (Islamic praying) regularly. Participants advised some clients to meet members of their clergy because they often asked about, or were deeply interested in theology, i.e. bible interpretation, in the counselling process. On the other hand, some participants did not recommend anything related to SR because of several reasons: the client had a different religion, SR was perceived as not part of conventional psychotherapy, they had insufficient knowledge and research articles about SRT, and they did not have personal experience of SRT especially for ruqyah. Many clients quite often inquired the participants whether they knew any SRH, particularly ruqyah practitioners. Most of the participants did not have such networks or knew SRH so they never made referrals. Participant 41 even asked their clients to search for SRH on Google. Additionally, some participants mentioned that they could not refer clients to SRH because it was outside the PHC and would not be covered by the insurance. However, some participants did a non-clinical referral to clergy members or to more senior CPs with more advanced religious knowledge and SRT skills. In addition, Participant 15 referred some 
clients to her partner who studied theology because clients wanted to discuss religious teachings more deeply.

Improving SRT knowledge and skills. Participants tried to develop their SRT knowledge and skills from various sources: mass media, TV programs, workshops, mentoring with senior CPs, and assisting in a clinical trial of zikir (Islamic chanting) therapy. In addition, SRT was also taught at Islamic universities in three ways as told by some Muslim participants. First, SR content was blended in the introduction to conventional psychotherapy course. Second, students were taught an Islamic Psychology course which also covered Islamic healing concepts. Lastly, practical courses about Islamic religious therapy techniques were taught only at the master's level. Participants also explained that some of their academics discouraged students from learning or practising yoga and meditation since these two methods are rooted in other religions, Hinduism and Buddhism respectively. Despite this prohibition, participants explained that CPs need to know the core concepts of other religions. This knowledge is essential so that CPs can better understand their clients from different religions and faith. Participants added having cultural awareness and competency is a must for CPs because their clients were not from one religion or belief system only.

\section{Shaping holistic mental health services}

Despite unfavourable attitudes towards spiritual healers, participants agreed that aspects of SR could not be isolated from psychological services. Participants perceived that the integration of SR into clinical practice would make mental health services more accepted in Indonesia. Therefore, this last theme presents two sub-themes of participants' efforts towards creating, and suggestions for creating, sustainable holistic mental health services. 
492 Applying a client-centred focus. Participants may have their own spirituality and religious

493

494

495

496

497

498

499

500

501

502

503

504

505

506

507

508

509

510

511

512

513

514

515 views and may disagree with a client's choice or views. Participants emphasised, however, that they had to behave as professional helpers by respecting their clients' beliefs. For example, participants respected their clients' rights and decisions when they or their family members stopped coming to conventional health services and went only to SRH. This clientcentred focus is important because participants did not want to make clients feel judged or blamed, which may break trust with the clients and obstruct their psychotherapy process. Moreover, participants perceived what clients or their family did, by visiting SRHs, as an effort that needed to be appreciated. Additionally, participants explained that clients who stop seeing SRH because of being forced to by their CP, may lose social support from family members because it might be perceived that the client does not trust their family anymore. Therefore, participants usually educated clients and their families about mental disorders and holistic mental health treatments from a conventional medicine perspective. For example, Participant 16 informed their clients in rural areas, who believed mental disorders were caused by supernatural forces, that any supernatural forces were in fact negative energy which was damaging clients' bodies and minds. Hence, clients needed some form of stronger energy to defeat it and they could increase their energy by coming to conventional health services, doing psychotherapy and physical exercise, and complement these treatments by visiting SRHs for spiritual support. Participants also frequently encouraged clients and their families to do self-critical thinking. Some topics and questions that often raised were whether there were any health benefits or side effects from visiting SRHs and what the cost had been. Clients and their families usually gained insight from these questions, including that not much progress had been made after visiting a SRH multiple times and that these visits cost them a lot because the insurance did not cover this treatment. After realising these facts, 
clients and their families mostly came to conventional health services regularly and visited SRHs just for spiritual support.

The client-centred focus was also applied when participants observed their clients' problems. Participants said that SRT was mostly effective for elderly clients because their problems were generally rooted in spiritual issues such as questioning their meaning of life and fear of dying. Participants also noticed that SRT was more suitable for clients with low education levels and socio-economic status (SES) compared with clients from high education level and socio-economic backgrounds. For example, murratal (reciting Quran in semisinging style) was recommended to clients with low SES as part of relaxation techniques and listening to classical music which they were not familiar with. Participants also considered integrating SRT for clients with formal religious education backgrounds such as those graduated from pondok pesantren (Islamic boarding schools, typically for Year 7 to 12) because clients usually would then be more engaged with the psychotherapy process.

\section{Collaboration with multiple stakeholders. Participants suggested that a credible SRH} should be considered an ally rather than a rival with whom to develop beneficial collaborative work. Participants positively welcomed this potential collaboration because they had experiences where some clients visited PHC because they were requested by the SRH that clients met before coming to the PHC. Participants perceived this phenomenon as an opportunity to improve mental health services in Indonesia and to make them more holistic. The collaboration might start from dialogue and mutual understanding, especially about mental disorders and treatments from a combined biopsycho-social-spiritual perspective. Moreover, CPs should have communication with trustworthy SRHs to avoid contradictive or overlapping SR activities if they work together in the future. 
540 Participants considered that they could not establish holistic mental health services by

541 themselves. Therefore, collaboration should also be developed between several stakeholders.

542 First, the collaboration could be made with other health professionals, particularly physicians,

543 where CPs may inform them if clients are taking herbal remedies from SRHs in order that

544 any adverse-drug-reaction can be avoided. The second proposal was to collaborate with kader

545 kesehatan jiwa (mental health cadres/MHC, volunteers who act as mental health sentinels in

546 the community) and local leaders. Participants explained that these stakeholders are more

547 respected and trusted than CPs by people in the area so that CPs should involve themselves

548 when organising events to educate the community about mental disorders. Also, local leaders

549 should know credible SRHs that CPs can invite for collaboration. Lastly, participants advised

550 making collaborations with schools because some child clients visited PHC after the teacher

551 persuaded the children's parents to not only go to SRH but also to meet a CP.

552

553

554

555

556

557

558

\section{Discussion}

This study aimed to explore how CPs address aspects of SR in Indonesia. The particular aspects examined were their attitudes towards and experiences of SR in a mental health context. In answering this question, participants initially explained the reasons why clients often visited SRHs first before seeking help from conventional health services when a client's condition became worse. It was found that the client's decision to visit a SRH was influenced by complex sociocultural factors as is often found in psychiatric care in Asia (Chaudhry, 2008; Kalra et al., 2015). Additionally, the finding from this study supported the results of another study into SR integration in palliative care in Indonesia that the family is often deeply involved in a client's health treatment (Rochmawati et al., 2018).

\section{Part of culture and belief}

The current study found that SR cannot be separated from mental health treatment because it is part of culture and belief in Indonesia. SR in Indonesia is not only embracing 
565 one official religion and obeying God; it is also one of rich heritage of traditions from local

566 belief systems that have existed since before the eighth century (Ropi, 2017). On the positive

567 side, SR was perceived to help improving client's mental health and well-being as found in

568 previous studies (Brown et al., 2013; Ramakrishnan et al., 2015). Nevertheless, at the same

569 time, SR might also hinder conventional mental health treatment because people believe

570 more in SRHs than in the treatments offered by CPs. The psychology profession may also be

571 misinterpreted because of people's unfamiliarity with it.

Moreover, participants explained that during the first year of being placed at the PHC

(initially in 2006-2007), they only had a few clients because the stigma surrounding mental

disorders was so intense, a phenomenon found across many Asian contexts (Chaudhry, 2008;

Javed, 2015). People in the city nowadays have a greater level of understanding of mental

disorders and the psychology profession, but the case is less with people in remote areas.

Therefore, people who visited SRHs mostly came from rural areas or villages, as found in a previous study in Bali (Suryani et al., 2011). This two-sided coin perspective was similar with the CPs' views on SR integration in psychotherapy in South Africa (Brown et al., 2013). genuinely integrated aspects of SR into their clinical practice. Additionally, it was difficult for participants in the current study to hide their religious identity when meeting clients because, for example, the majority of them were wearing hijab and others had Christian names. Some participants felt a dilemma because they were afraid to break a professional oath of neutrality. The same feeling was also found among Muslim CPs in South Africa where Muslim clients preferred having sessions with them rather than with Christian CPs 
590 this study highly recommended SR integration to be addressed by psychology faculties and 591 professional organisations, as was also suggested in previous studies among CPs in the UK 592 (Crossley \& Salter, 2005) and the USA (Shafranske \& Cummings, 2013).

593

594

595

596

597

598

599

600

601

602

603

604

605

606

607

608

609

610

611

612

613

614

\section{Attitudes towards spiritual-religious therapy}

Participants showed mixed attitudes towards SRT, particularly regarding its

effectiveness. These diverse attitudes might be due to different interpretations of religious teachings among them, even from those having the same religion. For example, Participant 29 supported her client to do ruqyah (Islamic exorcism) because she interpreted it as part of Islamic healing techniques. On the other hand, Participant 22 discouraged her client from doing ruqyah because she did not believe in it and ruqyah is a debatable issue among Islamic scholars. This contradiction reinforced previous studies which found that spirituality is wider, more unique and personal than religiosity (Chaudhry, 2008; Crossley \& Salter, 2005; Harris et al., 2016). Additionally, the difficulty in finding scientific evidence with a solid methodology to support SRT effectiveness may also constrain participants in completely believing in SRT efficacy, an issues which was also found among physicians and psychiatrists in the USA (Lawrence, Rasinski, Yoon, \& Curlin, 2014). However, participants in the current study did not prohibit clients from visiting SRHs, but rather encouraged them to come to conventional health services as well. This may be because the holistic mental health approach is advocated for by participants, similar with African CPs (Brown et al., 2013) who believe human health consists of physical, psychological, social, and spiritual aspects. This study found that participants showed favourable attitudes towards religious healers, but less favourable attitudes towards spiritual healers, which parallels a study among British CPs (Crossley \& Salter, 2005) and American psychiatrists (Lawrence et al., 2014). The main reason was that the methods given to the clients were perceived as irrational and risky, that would be eventually worsening a client's condition. In addition, Muslim 
participants in this study said that some of those methods deviated from Islamic teachings so that they had to warn clients as fellow Muslims. Muslim CPs in South Africa also alerted clients about behaviours which deviated from Islamic teaching because they were afraid God would punish them if they did not remind their fellow Muslims (Patel \& Shikongo, 2006). Catholic counsellors in Czech also felt that God was overseeing their counselling process (Motalová \& Řiháček, 2016). However, rather than warning client directly, they prayed to God to forgive their clients' sins. These results showed that SR personal values might affect mental health professionals' actions in their services.

\section{Experiences related to SR}

Though SR was used in participants' professional practice, they were still able to distinguish themselves from SRHs by their adherence to conventional psychotherapy procedures, as suggested in previous studies (Crossley \& Salter, 2005; Motalová \& Řiháček, 2016). It was discovered that participants felt more comfortable to do implicit rather than explicit SR integration which parallels with South African CPs (Brown et al., 2013). This means that participants would integrate SR activities as part of their psychotherapy but would not overtly practice it with clients in sessions. Additionally, participants substantially highlighted the importance of assessing the client's SR before integrating SR into the psychotherapy, as suggested in practical guidelines for psychologists and counsellors in the USA (Barnett \& Johnson, 2011; Matise, Ratcliff, \& Mosci, 2018). Participants in the current study also highlighted their confusion about a double-role where some of their colleagues and senior CPs acted as both psychologists and as members of the clergy. This multiple relationship issue was also questioned by American psychologists (Barnett \& Johnson, 2011, p. 156) as illustrated, "Where does the role of mental health practitioner end and the role of religious or spiritual leader begin?". The proposed alternative solution by participants paralleled one previous study (Shafranske \& Cummings, 2013) which was that clear 
regulations or a SOP of SR integration in psychological therapy should be created by professional organisations.

While all participants acknowledged that SR is important in Indonesian mental health treatment, not all of them integrated it into their practice. As similarly found among British and German CPs (Crossley \& Salter, 2005; Hofmann \& Walach, 2011), these participants felt uncomfortable to raise SR in their psychotherapy or uncertain about the related ethical issues. The majority of participants also did not recommend or refer clients to SRHs and asked clients to search for such persons by themselves. It may be potentially risky for clients to find SRHs independently because they could meet incompetent SRHs and worsen their condition. Therefore, mental health professionals should establish a network with local religious organisations or a credible spiritual healer association so that they can advise clients about which SRH to consult, as suggested by psychiatrists in the USA (Lawrence et al., 2014). Previous studies in Texas, USA, found that SR education or courses with SR content were incorporated significantly higher in nursing, counselling, and marriage-family therapy than in professional psychology education programs (Oxhandler \& Parrish, 2018). Moreover, more than $80 \%$ of German CPs reported receiving no SR education in their professional training (Hofmann \& Walach, 2011). In the current study it was discovered that the majority of participants from Islamic universities were intensively taught about religious therapy especially within an Islamic teaching context. Similar with recommendations from one previous study (Shafranske \& Cummings, 2013), education about SR in psychology curricula can be delivered in three methods: incorporating SR into existing psychology courses; training in SR and cultural sensitivity in clinical internships; and delivering special workshops about religious therapy. However, professional organisations must supervise the curriculum to prevent creating dissimilar competencies among CPs. 

more about and practising some complementary-alternative medicine (CAM) methods that are rooted in other religions, especially yoga. This might be because the Majelis Ulama Indonesia (MUI, Islamic Council of Indonesia) prohibited Muslims from practising yoga in 2008 (Ramstedt, 2010). The prohibition was not the first because, back in 1984, the Islamic Council of Singapore had issued a fatwa (non-binding Islamic legal pronouncement) to forbid Singaporean Muslims from practising it; followed by Egypt (2004) and Malaysia (2008). The reason was yoga was perceived to contain Hindu elements that might damage the foundation of the Islamic creed. The condemnation of yoga practice also happened in American Christian society because it was perceived as a demonic practice and spiritual invasion of Christianity (Miller, 2008). Despite the discouragement, participants in the present study expressed their interest in learning other religions' basic teachings as found among Indonesian palliative care staff (Rochmawati et al., 2018) because their clients were from a variety of religious backgrounds.

In achieving holistic mental health services, participants realised they have to work collaboratively with multiple stakeholders. For example, the presence of MHC in every local area should be an additional advantage in the collaboration because they are more respected and trusted than CPs (Miller, 2012). Some participants emphasised that SRHs should be perceived as an ally, as was also substantially advised in previous studies among American psychologists for religious counselling and spiritual support if needed by clients (Delaney et al., 2007; Shafranske \& Cummings, 2013). However, the boundaries and communication between professions have to be made clear to avoid unethical and overlapping practices. Additionally, through consistent education about holistic mental health treatments, stigma towards mental disorders may be decreased and encourage people to visit conventional health centres beside SRHs, as was found in Bali (Suryani et al., 2011). Spiritual healers in 
689 Indonesia were called by many names such as orang pintar (the wise person) and dukun 690 (shaman). Therefore, SRHs may be encouraged to educate their clients that SRT is part of 691 holistic mental health treatments and not like 'a spiritual pill' which will instantly solve 692 clients’ problems (Motalová \& Řiháček, 2016).

693

694

695

696

697

698

699

700

701

702

703

704

705

706

707

708

709

710

711

712

713

\section{Limitations and suggestions}

Although this novel study provided rich findings from 43 CPs, three limitations should be considered. First, the majority of participants were Muslims and all interviews were conducted in Java. Thus, the results of the current study might not be entirely applicable in different sociocultural settings and locations in Indonesia. Further exploration among CPs from diverse backgrounds is needed, for example, CPs in Bali where Hinduism is the dominant religion. In the international context, it might be interesting to conduct similar explorations among CPs in other nations with similar cultural influences, such as Malaysia and Brunei, and compare the results. Second, there was only one male CP interviewed. Males CP might address SR in their practice differently than females CP. Consequently, attitudes towards and experience of SR in a mental health context among males CP may be explored in the future study. Third, definitions of spirituality and religiosity were not separated nor given to the participants due to the nature of this exploratory study. Therefore, participants might interpret spirituality and religiosity as overlapping or interchangeable. Future research is needed to investigate CPs' understanding and experience of spirituality and religiosity both in their personal and professional lives.

\section{Implications for clinical practice and training}

Findings from the interviews may be used to improve clinical psychology practice and training. First, professional organisations should establish regulations and standard operating 
714 procedures to integrate SR or conduct spiritual-religious therapy in conventional

715

716

717

718

719

720

721

722

psychotherapy. Guidelines for SR integration from American psychologists and counsellors (Barnett \& Johnson, 2011; Matise et al., 2018) might be adapted according to participants' need and culture in Indonesia. Professional organisations should also monitor CPs who play a double-role as a member of the clergy and as a mental health professional to prevent unethical practice. Second, psychology faculties should educate and train their students about SR, and how to address and integrate it in their future practice as CPs. Professional organisations need to oversee this education to ensure that every student will have the same SR competency when they graduate. Lastly, CPs should proactively build networks with local religious organisations or credible spiritual healers for discussion about SR or when a client needs a referral.

\section{Conclusion}

The most notable finding from this novel qualitative study was represented by an excerpt from Participant 12 in the title, "Doing my profession is also part of worship".

Participants perceived SR as part of culture and belief among Indonesian people, including CPs and mental health treatment clients. Participants genuinely acknowledged that they were not able to completely detach SR from their professional practice. However, participants also pointed out that they were different with SRHs and favourably welcomed future collaboration with credible SRHs. This positive attitude embodied a holistic care approach that recognises the diverse biopsycho-social-spiritual needs of clients. It also supports a previous study among Germany CPs (Hofmann \& Walach, 2011, p. 187) that highlighted, "psychology as a discipline is secular, but psychotherapists as individuals often are not." Education of SR in psychology curricula and the regulation of SR integration into conventional psychotherapy are needed to achieve this holistic mental health services in Indonesia. Moreover, participants highly advised CPs to collaborate with multiple sectors, i.e. other health professionals, because they cannot work alone. 
739

740

741

742

743

744

745

746

747

748

749

750

751

752

753

754

755

756

757

758

759

\section{Author's short-bio}

Andrian Liem is a $\mathrm{PhD}$ candidate (supervised by $\mathrm{A} / \mathrm{Prof}$ Peter $\mathrm{A}$. Newcombe, $\mathrm{PhD}$ and Annie E. Pohlman, PhD) and a casual tutor at the School of Psychology, the University of Queensland, Australia. Previously, he finished professional psychology training in clinical psychology and Master of Psychology from the University of Gadjah Mada, Indonesia. His research interests include clinical-health psychology, indigenous and cultural psychology, cultural competency, gender and sexuality, drug abuse, HIV-AIDS, and interfaith dialogue. Andrian designed the research, collected and analysed data (with guidance from Annie E. Pohlman as his supervisor), drafted and finalised the manuscript.

\section{Acknowledgments}

Data of this study is part of the author's doctoral thesis on CAM among clinical psychologists in Indonesia. The author thanks the Indonesian Clinical Psychology Association (IPK HIMPSI) for the permission to collect data and psychologists who participated in the interviews. The author thanks Annie E. Pohlman, PhD for the invaluable feedback and discussion.

\section{Conflict of interest}

No conflict of interest to declare.

\section{Funding}

The author is supported by Indonesia Endowment Fund for Education Scholarship (LPDP RI) for the doctoral degree at the University of Queensland (20150122082410). The views expressed are the author's own. 
Table 1 Comparison of uses of SR by CP and SRH

\begin{tabular}{|c|c|c|}
\hline Aspect & $\mathbf{C P}$ & SRH \\
\hline $\begin{array}{l}\text { Assessment and } \\
\text { treatment plan }\end{array}$ & Structured and standardised & Not clear \\
\hline Intervention & $\begin{array}{l}\text { - Simple and basic techniques } \\
\text { based on scientific evidence } \\
\text { - SR supports conventional } \\
\text { psychotherapy and is } \\
\text { integrated only if needed } \\
\text { - The reason and psychological } \\
\text { benefit from the intervention } \\
\text { are explained scientifically }\end{array}$ & $\begin{array}{l}\text { - Complex and advanced rituals } \\
\text { or teaching based on faith } \\
\text { - SR is the only technique used } \\
\text { for whatever the problem is } \\
\text { - The reasons and benefits of the } \\
\text { intervention are explained } \\
\text { using a theological approach } \\
\text { or not explained at all }\end{array}$ \\
\hline $\begin{array}{l}\text { Communication } \\
\text { style }\end{array}$ & Use micro-counselling skills & Dogmatic and doctrinal \\
\hline
\end{tabular}

761 
762

763

764

765

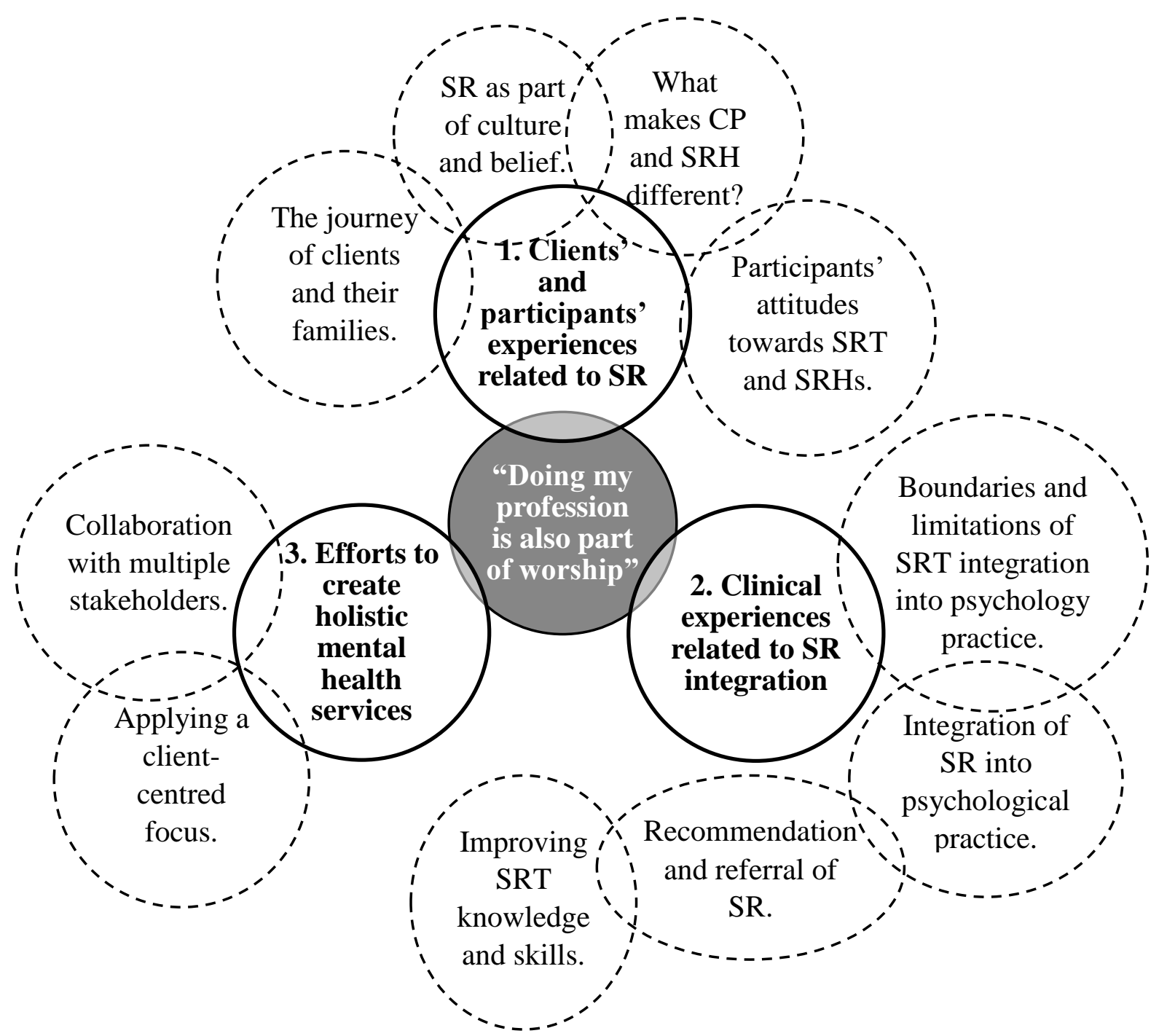

Figure $1 \mathrm{~A}$ visual map of themes and sub-themes

Notes. $\mathrm{CP}=$ Clinical psychologist $\mathrm{SR}=$ Spirituality and religion; $\mathrm{SRHs}=$ Spiritual-religious healers; SRT $=$ Spiritual-religious therapy. 
Reasons for going to SRHs (according to participants):

- Part of culture and belief;

- Avoids stigma associated with mental disorders;

- Client's misunderstanding of CP's profession, i.e. CPs are only for severe mental disorders;

- Insufficient knowledge of mental disorders and treatment from a conventional medicine perspective;

- Client's beliefs, i.e. cursed or redemption from sin;

- Perception of SRHs, particularly members of the clergy, as close to God so clients expect that clergy can forward their messages on to God;

- Perception of SRT conducted by SRH as safer than conventional medicine;

- Trust in SRHs more than conventional health practitioners;

- More convenient distance and flexible practice hours with SRHs than conventional health services;

- Only a single and short session, and no referral which saves time;

- Potential financial loss due to the location of PHC and lengthy time of conventional medicine administrative work and medical process;

- Encouragement by family members, relatives, or neighbours; and

- Advertisement of SRHs on the street.

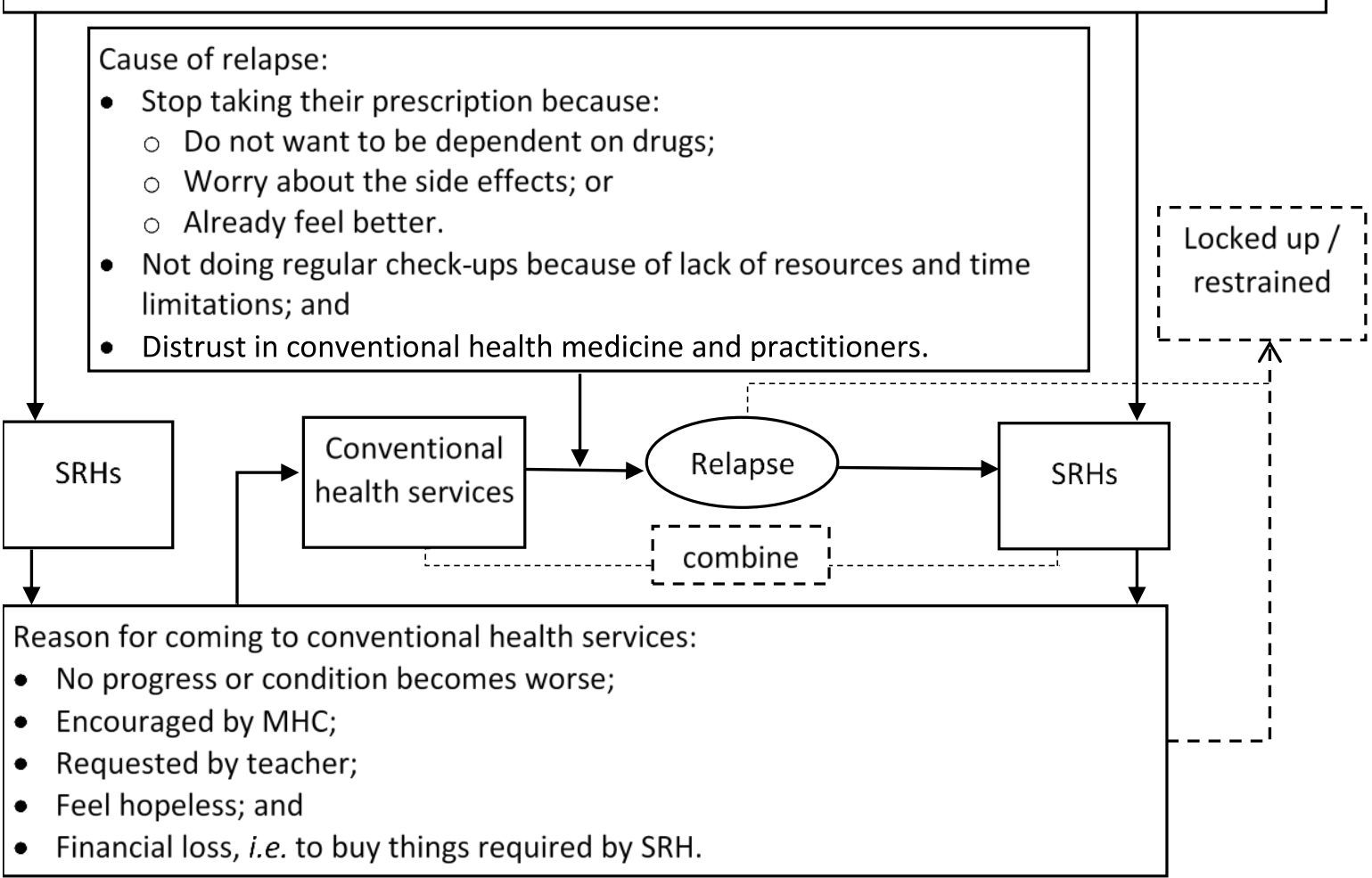

Figure 2 The pattern of client's behaviour in visiting SRHs and conventional health services Notes. $\mathrm{MHC}=$ Mental health cadre (volunteers who act as mental health sentinels in the community); SRHs = Spiritual-religious healers. 


\section{References}

782

783

784

785

786

787

788

789

790

791

792

793

794

795

796

797

798

799

800

801

802

803

804

805

806

807

808

809

810

811

812

813

Bantjes, \& Van Ommen. (2008). The development and utilisation of a suicide risk assessment interview schedule. South African Journal of Psychology, 38, 391-411.

Barnett, \& Johnson. (2011). Integrating spirituality and religion into psychotherapy: Persistent dilemmas, ethical issues, and a proposed decision-making process. Ethics \& Behavior, 21, 147-164.

Braun, \& Clarke. (2006). Using thematic analysis in psychology. Qualitative Research in Psychology, 3, 77-101.

Brown, Elkonin, \& Naicker. (2013). The use of religion and spirituality in psychotherapy: Enablers and barriers. Journal of Religion and Health, 52, 1131-1146. doi:10.1007/s10943-011-9551-z

Chaudhry. (2008). Psychiatric care in Asia: Spirtuality and religious connotations. International Review of Psychiatry, 20, 477-483.

Crossley, \& Salter. (2005). A question of finding harmony: A grounded theory study of clinical psychologists' experience of addressing spiritual beliefs in therapy. Psychology and Psychotherapy: Theory, Research and Practice, 78, 295-313.

Delaney, Miller, \& Bisonó. (2007). Religiosity and spirituality among psychologists: A survey of clinician members of the American Psychological Association. Professional Psychology: Research and Practice, 38, 538.

Dikko. (2016). Establishing construct validity and reliability: Pilot testing of a qualitative interview for research in takaful (Islamic insurance). The Qualitative Report, 21, 521-528.

Frazier, \& Hansen. (2009). Religious/spiritual psychotherapy behaviors: Do we do what we believe to be important? Professional Psychology: Research and Practice, 40, 81.

Guest, MacQueen, \& Namey. (2012). Applied thematic analysis. Los Angeles: Sage Publications.

Harris, Randolph, \& Gordon. (2016). What do clients want? Assessing spiritual needs in counseling: A literature review. Spirituality in Clinical Practice, 3, 250-275.

Hofmann, \& Walach. (2011). Spirituality and religiosity in psychotherapy-A representative survey among German psychotherapists. Psychotherapy Research, 21, 179-192.

Javed. (2015). Programs of mental health and policies in South Asia: Origin and current status. In J. K. Trivedi \& A. Tripathi (Eds.), Mental Health in South Asia: Ethics, Resources, Programs and Legislation (pp. 81-94). Dordrecht: Springer Netherlands.

Kalra, Shah, Deakin, \& Bhugra. (2015). Ethical handling of religious and spiritual issues: South Asian perspective. In J. K. Trivedi \& A. Tripathi (Eds.), Mental Health in South Asia: Ethics, Resources, Programs and Legislation (pp. 35-47). Dordrecht: Springer Netherlands. 
Kementerian Kesehatan RI. (2013). Riset kesehatan dasar 2013. Jakarta, Indonesia: Badan Penelitian dan Pengembangan Kesehatan Departemen Kesehatan Republik Indonesia.

Lawrence, Rasinski, Yoon, \& Curlin. (2014). Primary care physicians' and psychiatrists' willingness to refer to religious mental health providers. International Journal of Social Psychiatry, 60, 627636.

Liem. (2018). Interview schedule development for a sequential explanatory mixed method design: Complementary-alternative medicine (CAM) study among Indonesian psychologists. International Journal of Social Research Methodology, 21, 513-525. doi:10.1080/13645579.2018.1434864

Liem, \& Newcombe. (2019). Knowledge, attitudes, and usage of complementary-alternative medicine (CAM): A national survey of clinical psychologists in Indonesia. Current Psychology, Online first, 1-11. doi:10.1007/s12144-019-00290-1

Liem, \& Rahmawati. (2017). The meaning of complementary, alternative and traditional medicine among the Indonesian psychology community: A pilot study. Journal of Integrative Medicine, 15, 288-294.

Matise, Ratcliff, \& Mosci. (2018). A Working Model for the Integration of Spirituality in Counseling. Journal of Spirituality in Mental Health, 20, 27-50.

Miller. (2008). The yoga boom: A call for Christian discernment Part 3: Toward a comprehensive Christian response. Christian Research Journal, 31, 1-12.

Miller. (2012). Who needs psychiatrists? Science Magazine, 335, 194-1298.

Motalová, \& Řiháček. (2016). Religiosity gap reversed: How religious counsellors' belief system presents when working with clients in a non-religious environment. British Journal of Guidance and Counselling, 44, 277-288.

Oxhandler, \& Parrish. (2018). Integrating clients' religion/spirituality in clinical practice: A comparison among social workers, psychologists, counselors, marriage and family therapists, and nurses. Journal of Clinical Psychology, 74, 680-694. doi:10.1002/jclp.22539

Passalacqua, \& Cervantes. (2008). Understanding gender and culture within the context of spirituality: Implications for counselors. Counseling and Values, 52, 224-239.

Patel, \& Shikongo. (2006). Handling spirituality/religion in professional training: Experiences of a sample of Muslim psychology students. Journal of Religion and Health, 45, 93-112. doi:10.1007/s10943-005-9009-2

Ramakrishnan, Karimah, Kuntaman, Shukla, Ansari, Rao, ... Murthy. (2015). Religious/spiritual characteristics of Indian and Indonesian physicians and their acceptance of spirituality in 
847

848

849

850

851

852

853

854

855

856

857

858

859

860

861

862

863

864

865

866

867

868

869

870

871

872

873

874

health care: A cross-cultural comparison. Journal of Religion and Health, 54, 649-663. doi:10.1007/s10943-014-9906-3

Ramstedt. (2010). A Fatwa against yoga: Mitigating conflict in the face of increasing fundamentalism in Indonesia. Journal of Inter-Religious Dialogue, 5, 22-31.

Rochmawati, Wiechula, \& Cameron. (2018). Centrality of spirituality/religion in the culture of palliative care service in Indonesia: An ethnographic study. Nursing and Health Sciences, 20, 231-237.

Ropi. (2017). Religion and Regulation in Indonesia: Springer Verlag.

Setiyawati, Blashki, Wraith, Colucci, \& Minas. (2014). Indonesian experts' perspectives on a curriculum for psychologists working in primary health care in Indonesia. Health Psychology and Behavioral Medicine: An Open Access Journal, 2, 623-639. doi:10.1080/21642850.2014.912946

Shafranske, \& Cummings. (2013). Religious and spiritual beliefs, affiliations, and practices of psychologists.

Shafranske, \& Malony. (1990). Clinical psychologists' religious and spiritual orientations and their practice of psychotherapy. Psychotherapy: Theory, Research, Practice, Training, 27, 72.

Smith, \& Wu. (2012). Nurses' beliefs, experiences and practice regarding complementary and alternative medicine in Taiwan. Journal of Clinical Nursing, 21, 2659-2667.

Suryani, Lesmana, \& Tiliopoulos. (2011). Treating the untreated: applying a community-based, culturally sensitive psychiatric intervention to confined and physically restrained mentally ill individuals in Bali, Indonesia. European Archives of Psychiatry and Clinical Neuroscience, 261, 140-144. doi:10.1007/s00406-011-0238-y

Tong, Sainsbury, \& Craig. (2007). Consolidated criteria for reporting qualitative research (COREQ): A 32-item checklist for interviews and focus groups. International Journal for Quality in Health Care, 19, 349-357.

Vaismoradi, Turunen, \& Bondas. (2013). Content analysis and thematic analysis: Implications for conducting a qualitative descriptive study. Nursing \& health sciences, 15, 398-405. 